\title{
Lucas numbers of the form $\left(\begin{array}{l}2^{t} \\ k\end{array}\right)$
}

\author{
NuRettin Irmak ANd LÁszló Szalay \\ To the memory of Professor József Závoti
}

\begin{abstract}
Let $L_{m}$ denote the $m^{\text {th }}$ Lucas number. We show that the solutions to the diophantine equation $\left(\begin{array}{c}2^{t} \\ k\end{array}\right)=L_{m}$, in non-negative integers $t, k \leq 2^{t-1}$, and $m$, are $(t, k, m)=(1,1,0),(2,1,3)$, and $(a, 0,1)$ with non-negative integers $a$.
\end{abstract}

\section{Introduction}

As usual, the sequence of Lucas numbers is defined by $L_{0}=2, L_{1}=1$, and

$$
L_{m}=L_{m-1}+L_{m-2}, \quad m \geq 2 .
$$

This sequence is known as the associate of Fibonacci sequence.

Now we present a short historical background related to the title problem. The occurrence of figurate numbers in linear recurrences has had a very extensive literature. The first challenging result is due to Cohn $[1,2]$, and independently to Wyler [18], who proved that the square Fibonacci numbers are $F_{0}=0, F_{1}=F_{2}=1$ and $F_{12}=144$. Focusing only on the occurrence of binomial coefficients in binary recurrences, first we mention that Ming [11] proved a conjecture of Hoggatt [5]. Namely, he showed that $F_{0}=0, F_{1}=$ $F_{2}=1, F_{4}=3, F_{8}=21$ and $F_{10}=55$ are the only triangular Fibonacci numbers, further $L_{1}=1, L_{2}=3$ and $L_{18}=5778$ are the only Lucas triangular numbers [12]. Note that the triangular number $t_{n-1}=(n-1) n / 2$ is equal to the binomial coefficient $\left(\begin{array}{l}n \\ 2\end{array}\right)$. Therefore, it seems natural to search the binomial coefficients $\left(\begin{array}{l}n \\ k\end{array}\right)$ in certain recurrences. Special cases of this question were handled by several authors, see, for example, [3].

Consider the binary recurrence $U_{m}=A U_{m-1}+B U_{m-2}$ with arbitrary initial values $U_{0}$ and $U_{1}$. If $\left\{V_{m}\right\}$ is the associate of $\left\{U_{m}\right\}$ (i.e., the two

Received April 10, 2018.

2010 Mathematics Subject Classification. 11B39; 11D72.

Key words and phrases. Lucas number; binomial coefficient; diophantine equation. http://dx.doi.org/10.12697/ACUTM.2019.23.06 
sequences have the same recurrence rule, further $V_{0}=2 U_{1}-A U_{0}$ and $V_{1}=$ $\left.A U_{1}+2 B U_{0}\right)$, then their terms satisfy

$$
V_{n}^{2}-D U_{n}^{2}=4 C(-B)^{n},
$$

where $D=A^{2}+4 B$ and $C=U_{1}^{2}-A U_{0} U_{1}-B U_{0}^{2}$.

Fix $|B|=1$. Replacing either $V_{n}$ or $U_{n}$ by $\left(\begin{array}{l}n \\ 2\end{array}\right)$, (1.1) leads to the superelliptic equation

$$
y^{2}=D n^{4}-2 D n^{3}+D n^{2} \pm 16 C .
$$

The Magma [10] procedure IntegralQuarticPoints () may solve this equation. Hence if the lower index $k$ is 2 in $\left(\begin{array}{l}n \\ k\end{array}\right)$, then we are able to handle the problem for certain binary recurrences.

For the lower index $k=3$ an algorithm was given in [16] to solve the equations

$$
U_{m}=\left(\begin{array}{l}
n \\
3
\end{array}\right) \quad \text { and } \quad V_{m}=\left(\begin{array}{l}
n \\
3
\end{array}\right),
$$

with the conditions $D>0$, and $U_{0}=0, U_{1}=1$ (and $|B|=1$ ). Illustrating the algorithm, all integer solutions to the equations

$$
F_{m}=\left(\begin{array}{l}
n \\
3
\end{array}\right), \quad L_{m}=\left(\begin{array}{l}
n \\
3
\end{array}\right) \quad \text { and } \quad P_{m}=\left(\begin{array}{l}
n \\
3
\end{array}\right)
$$

were given in [16]. Here $P_{m}$ is a term of the Pell sequence.

Later, Szalay [15] treated the equations $F_{m}=\left(\begin{array}{c}n \\ 4\end{array}\right), L_{m}=\left(\begin{array}{l}n \\ 4\end{array}\right)$, and Kovács [6] solved the analogous equation $P_{m}=\left(\begin{array}{l}n \\ 4\end{array}\right)$. The more complicated problem $L_{m}=\left(\begin{array}{l}n \\ 5\end{array}\right)$ was handled by Tengely [17].

In this paper, as a novelty, we do not fix the lower subscript $k$, but on the other hand we prescribe $n=2^{t}$ with unknown non-negative integer $t$. Hence, for the Lucas numbers we study the diophantine equation

$$
L_{m}=\left(\begin{array}{l}
2^{t} \\
k
\end{array}\right) .
$$

The complete description of the result is given by the following theorem.

Theorem 1. The solutions to the diophantine equation

$$
L_{m}=\left(\begin{array}{c}
2^{t} \\
k
\end{array}\right)
$$

in non-negative integers $t, k \leq 2^{t-1}$, and $m$ are

$$
(t, k, m)=(1,1,0),(2,1,3) \text { and }(a, 0,1)
$$

with non-negative integers a. 


\section{Auxiliary results}

Assume that $p$ is a prime number. The $p$-adic order of a non-zero integer $n$ is the largest positive integer exponent $\nu$ of $p$ such that $p^{\nu}$ divides $n$. As usual, let $\nu$ be denoted by $\nu_{p}(n)$. For the integer $n=a_{0}+a_{1} p+a_{2} p^{2}+\cdots+$ $a_{d} p^{d},\left(0 \leq a_{i}<p\right)$, the digit sum function (in base $\left.p\right)$ is defined by

$$
s_{p}(n)=a_{0}+a_{1}+\cdots+a_{d} .
$$

In particular, Legendre [8] showed that

$$
\nu_{p}(n !)=\frac{n-s_{p}(n)}{p-1} .
$$

Lemma 1. Assume that $n$ and $k \leq 2^{n}-1$ are positive integers. Then

$$
\nu_{2}\left(\left(\begin{array}{c}
2^{n} \\
k
\end{array}\right)\right)=n-\nu_{2}(k) \text {. }
$$

Proof. It is clear that $\nu_{2}\left(2^{n}-j\right)=\nu_{2}(j)$ holds if $1 \leq j \leq 2^{n}-1$. Expanding the binomial coefficient we get

$$
\begin{aligned}
\nu_{2}\left(\left(\begin{array}{c}
2^{n} \\
k
\end{array}\right)\right) & =\nu_{2}\left(\frac{2^{n}\left(2^{n}-1\right) \ldots\left(2^{n}-k+1\right)}{k !}\right) \\
& =\nu_{2}\left(2^{n}\right)+\nu_{2}\left(2^{n}-1\right)+\ldots+\nu_{2}\left(2^{n}-(k-1)\right)-\nu_{2}(k !) \\
& =n+\nu_{2}((k-1) !)-\nu_{2}(k !)= \\
& =n-\nu_{2}(k) .
\end{aligned}
$$

We note that Kummer [7] derived a result from Legendre's theorem, which also proves the statement of the above lemma. Kummer's theorem says that the $p$-adic valuation of the binomial coefficient $\left(\begin{array}{l}a \\ b\end{array}\right)$ is equal to the number of carries when $a-b$ is added to $b$ in base $p$.

Citing [9], here we present the 2-adic order of the Lucas numbers.

Lemma 2. If $n \geq 0$ is an integer, then

$$
\nu_{2}\left(L_{n}\right)= \begin{cases}0, & \text { if } n \equiv 1,2 \quad(\bmod 3) \\ 1, & \text { if } n \equiv 0 \quad(\bmod 6), \\ 2, & \text { if } n \equiv 3 \quad(\bmod 6)\end{cases}
$$

Lemma 3. For any integer $n \geq 0$ we have $L_{n} \not \equiv 6(\bmod 8)$.

Proof. Consider the Lucas numbers modulo 8. The sequence becomes periodic with length 12 , and looking at the period, it leads immediately to the statement.

Lemma 4. A Lucas number $L_{n}$ with odd subscript $n$ is composed only of primes $p$ satisfying $p=2$ or $p \equiv \pm 1(\bmod 5)$. 
Proof. Although the proof comes straightaway from the well-know identity $L_{n}^{2}-5 F_{n}^{2}=4(-1)^{n}$, we simply refer to [13], last row of page 280 .

Lemma 5. Suppose that $a, b$ and $n$ are positive integers. Then

$$
\left(\begin{array}{c}
a n+b n \\
a n
\end{array}\right) \equiv 0 \bmod \left(\frac{b n+1}{\operatorname{gcd}(a, b n+1)}\right) .
$$

Proof. See Theorem 1.1 in [14].

Lemma 6. For $n \geq 1$ we have

$$
\left(\begin{array}{c}
2^{n+1} \\
2^{n}
\end{array}\right) \equiv 6 \quad(\bmod 8)
$$

Proof. It is obvious when $n=1$. Therefore we may assume $n \geq 2$.

In case of $p=2$ the Legendre formula (2.1) implies $\nu_{2}\left(2^{a} !\right)=2^{a}-1$. Subsequently,

$$
\nu_{2}\left(\left(\begin{array}{c}
2^{n+1} \\
2^{n}
\end{array}\right)\right)=\nu_{2}\left(\frac{2^{n+1} !}{\left(2^{n !}\right)^{2}}\right)=\nu_{2}\left(2^{n+1} !\right)-2 \nu_{2}\left(2^{n} !\right)=1,
$$

hence it is sufficient to consider the odd ingredients of the binomial coefficient in the lemma. To do that, observe that $h(a):=2^{a} ! / 2^{2^{a}-1}$ is an odd integer, and we need to see that $h(a) \equiv 3(\bmod 8)$ for $a \geq 2$. It is a direct consequence of Lemma 3.3 in the paper [4] by fixing there $p=2, b=3$, $t=1, i=0$, and $j=1$. Finally, $h(n+1) / h^{2}(n) \equiv 3 / 3^{2} \equiv 3(\bmod 8)$ proves the lemma.

\section{Proof of Theorem 1}

The statement is trivial for $k=0$, and we obtain the infinite family of solutions $(t, k, m)=(a, 0,1), a \geq 0$.

In the sequel, we assume $1 \leq k \leq 2^{t-1}$. Combining (1.2), Lemma 2, and Lemma 1, it provides

$$
j=t-\nu_{2}(k)
$$

where $j=0,1,2$. Thus, $t-j=\nu_{2}(k)$, and, consequently, $k=2^{t-j} s$ holds with some positive odd integer $s$. The condition $k=2^{t-j} s \leq 2^{t-1}$ is fulfilled only if $j=1$ or 2 , and in these cases $s=1$ necessarily holds. Hence $k=2^{t-j}$ $(j=1,2)$. For our convenience put $a=t-j$. Then $k=2^{a}$, and we distinguish two cases.

First let $j=1$. Now we have the equation

$$
L_{m}=\left(\begin{array}{c}
2^{a+1} \\
2^{a}
\end{array}\right)
$$

to solve. Taking both sides of this equation modulo 8 , Lemma 3 contradicts to Lemma 6 if $a \geq 1$. The remaining value $a=0$ leads to the solution $(t, k, m)=(1,1,0)$. 
Now let $j=2$. Clearly, by Lemma 2 we know that $m=6 \kappa+3$. We have

$$
L_{m}=\left(\begin{array}{c}
2^{a+2} \\
2^{a}
\end{array}\right),
$$

and first assume that $a$ is even. The case $a=0$ provides the solution $(t, k, m)=(2,1,3)$. Then we may suppose $a \geq 1$. Applying Lemma 5 , it yields that

$$
\left(\begin{array}{c}
2^{a+2} \\
2^{a}
\end{array}\right)=\left(\begin{array}{c}
3 \cdot 2^{a}+2^{a} \\
3 \cdot 2^{a}
\end{array}\right) \equiv 0 \bmod \left(\frac{2^{a}+1}{\operatorname{gcd}\left(3,2^{a}+1\right)}\right) .
$$

The parity of $a$ guarantees that the denominator of the modulus is 1, i.e., the modulus is $2^{a}+1$. Put $a=2 \ell$. Note that $\ell \geq 1$. Then we obtain

$$
L_{6 \kappa+3}=\left(\begin{array}{c}
4^{\ell+1} \\
4^{\ell}
\end{array}\right) \equiv 0 \quad\left(\bmod 4^{\ell}+1\right) .
$$

This gives that $4^{\ell}+1 \mid L_{6 \kappa+3}$. By Lemma 4 we have

$$
L_{6 \kappa+3}=p_{1} p_{2} \cdots p_{n},
$$

where $p_{i}$ are primes with $p_{i}=2$ or $p_{i} \equiv \pm 1(\bmod 5)$ for $1 \leq i \leq n$. Hence every prime factor $p_{j}$ of $4^{\ell}+1(\ell \geq 1)$ has the form $p_{j} \equiv \pm 1(\bmod 5)$. Thus,

$$
4^{\ell}+1=p_{i_{1}} p_{i_{2}} \cdots p_{i_{t}}
$$

follows with $t \leq n$. Now reduce (3.1) modulo 5 , and we arrive at a contradiction since $4^{\ell}+1 \equiv 0$ or $2(\bmod 5)$, and at the same time $p_{1} p_{2} \cdots p_{t} \equiv 1$ or $4(\bmod 5)$.

Assume that $a$ is odd, and let $a=2 \ell+1$ with a non-negative integer $\ell$. The case $\ell=0$ does not provide a solution to (1.2). So we may assume $\ell \geq 1$. Now we get

$$
\left(\begin{array}{c}
2^{a+2} \\
2^{a}
\end{array}\right)=\left(\begin{array}{c}
2^{a}+3 \cdot 2^{a} \\
2^{a}
\end{array}\right) \equiv 0 \quad\left(\bmod 3 \cdot 2^{a}+1\right)
$$

because trivially $\operatorname{gcd}\left(1,3 \cdot 2^{a}+1\right)=1$. Thus,

$$
3 \cdot 2^{a}+1=6 \cdot 4^{\ell}+1 \mid L_{6 \kappa+3},
$$

where the prime factors $p_{j}$ of $L_{6 \kappa+3}$ again satisfy $p_{j} \equiv \pm 1(\bmod 5)$. A modulo 5 consideration of $6 \cdot 4^{\ell}+1$, similarly to the previous case, leads to a contradiction.

The proof of Theorem 1 is complete.

\section{Acknowledgment}

The authors express his gratitude to the anonymous reviewer for the instructive suggestions. 


\section{References}

[1] J. H. E. Cohn, On square Fibonacci numbers, J. London Math. Soc. 39 (1964), 537540.

[2] J. H. E. Cohn, Square Fibonacci numbers, etc., Fibonacci Quart. 2 (1964), 109-113.

[3] A. Flammenkamp and F. Luca, Binomial coefficients and Lucas sequences, J. Number Theory 93 (2002), 246-284.

[4] L. Hajdu and A. Papp, On asymptotic density properties of the sequence $(n !)_{n=0}^{\infty}$, Acta Arithm. 184 (2018), 317-340

[5] V. E. Hoggatt, Problem 3, in: WA St. Univ. Conf. Number Theory, 1971, p. 225.

[6] T. Kovács, Combinatorial numbers in binary recurrences, Period. Math. Hungar. 58 1 (2009), 83-98.

[7] E. Kummer, Über die Ergänzungssätze zu den allgemeinen Reciprocitätsgesetzen, J. Reine Angew. Math. 44 (1852), 93-146.

[8] A. M. Legendre, Theorie des Nombres, I, Firmin Didot Freres, Paris, 1830.

[9] T. Lengyel, The order of the Fibonacci and Lucas numbers, Fibonacci Quart. 33 (1995), 234-239.

[10] MAGMA Handbook, http://magma.maths.usyd.edu.au/magma/handbook/

[11] L. Ming, On triangular Fibonacci numbers, Fibonacci Quart. 27 (1989), 98-108.

[12] L. Ming, On triangular Lucas numbers, in: Applications of Fibonacci numbers 4 (Winston-Salem, NC, 1990), Kluwer Acad. Publ., Dordrecht, 1991, pp. 231-240.

[13] P. Moree, Counting divisors of Lucas numbers, Pacific J. Math. 186 (1998), 267-284.

[14] Z. W. Sun, On divisibility of binomial coefficients, J. Aust. Math. Soc. 93 (2012), 189-201.

[15] L. Szalay, Some polynomial values binary recurrences, Rev. Col. Mat. 35 (2001), 99 106.

[16] L. Szalay, On the resolution of the equations $U_{n}=\left(\begin{array}{l}x \\ 3\end{array}\right)$ and $V_{n}=\left(\begin{array}{l}x \\ 3\end{array}\right)$, Fibonacci Quart. 40 (2002), 9-12.

[17] Sz. Tengely, On the diophantine equation $L_{n}=\left(\begin{array}{l}x \\ 5\end{array}\right)$, Publ. Math. Debrecen 79 (2011), $749-758$.

[18] O. Wyler, In the Fibonacci series $F_{1}=1, F_{2}=1, F_{n+1}=F_{n}+F_{n-1}$ the first, second and twelft terms are squares, Amer. Math. Monthly 71 (1964), 221-222.

NiĞde Ömer Halisdemir University, Art and Science Faculty, Mathematics Department, NiĞDE, TURKEY

E-mail address: nirmak@ohu.edu.tr, irmaknurettin@gmail.com

J. Selye University, Department of Mathematics and Informatics, Komarno, SLOVAKIA

E-mail address: laszlo.szalay.sopron@gmail.com 